\title{
Dual effects of FeNPs on maize growth and water use are actively mediated by arbuscular mycorrhizal fungus via rhizosphere interactions
}

yumiao Yang ${ }^{1}$, minha naseer ${ }^{1}$, ying $\mathrm{zhu}^{2}$, shuangguo $\mathrm{Zhu}^{1}$, baozhong Wang ${ }^{1}$, yibo Wang ${ }^{3}$, jing wang ${ }^{1}$, hao zhu ${ }^{1}$, wei wang ${ }^{1}$, li zhu ${ }^{1}$, yixia Gao $^{1}$, Hong-Yan Tao ${ }^{1}$, and youcai Xiong ${ }^{1}$

${ }^{1}$ Lanzhou University

${ }^{2}$ Gansu Academy of Sciences

${ }^{3}$ Tianshui Normal University

September 25, 2021

\begin{abstract}
Nanoscale zero-valent iron (FeNPs) can affect plant growth and water uptake positively or negatively. Yet, its mechanism is still unclear, particularly for differentiate functional roles of FeNPs in plant-soil interaction. To address this issue, we first confirmed 1.0 and $1.5 \mathrm{~g} \cdot \mathrm{kg}-1$ FeNPs as growth-promotion concentrations and $1.5 \mathrm{~g} \cdot \mathrm{kg}-1$ as optimal one, while $2.0 \mathrm{~g} \cdot \mathrm{kg}-1$ turned to restrict growth in maize seedlings, comparing with CK. When AM fungus (Funneliformis mosseae) was inoculated and cultured with FeNPs, 1.0 and $1.5 \mathrm{~g} \cdot \mathrm{kg}-1$ FeNPs further evidently enhanced water uptake and biomass accumulation $(\mathrm{P}<0.05)$. ), as affected by the activated AMF colonization carrier. Contrarily, FeNPs turned to promote AMF colonization and development, achieving greater iron translocation efficiency in mycorrhiza $(\mathrm{P}<0.05)$. Yet, $2.0 \mathrm{~g} \cdot \mathrm{kg}-1$ FeNPs restricted AMF infection in roots, a negative effect. To investigate the mycorrhizal-FeNPs symbiosis, scanning electron microscopy was used to scan the extraradical hyphae, showing that hyphae intercepted excessive iron nanoparticles, alleviating the blockage of root surface apertures and ultimately restoring root activity. Therefore, dual effects of FeNPs on maize physiology were actively mediated by AMF via rhizosphere interactions. The findings provided new insights into safe and efficient utilization of nanomaterials in agro-ecosystems.
\end{abstract}

\section{Hosted file}

Manuscript-20210908.doc available at https://authorea.com/users/436707/articles/538844dual-effects-of-fenps-on-maize-growth-and-water-use-are-actively-mediated-by-arbuscularmycorrhizal-fungus-via-rhizosphere-interactions

\section{Hosted file}

Figures-20210908(1).doc available at https://authorea.com/users/436707/articles/538844dual-effects-of-fenps-on-maize-growth-and-water-use-are-actively-mediated-by-arbuscularmycorrhizal-fungus-via-rhizosphere-interactions 